\title{
Hydrogen/deuterium exchange in interstellar ice analogs
}

\author{
A. Ratajczak ${ }^{1,2}$, E. Quirico ${ }^{2}$, A. Faure ${ }^{1}$, B. Schmitt ${ }^{2}$, and C. Ceccarelli ${ }^{1}$ \\ 1 Laboratoire d'Astrophysique de Grenoble, UMR 5571-CNRS, Université Joseph Fourier, Grenoble, France \\ e-mail: afaure@obs.ujf-grenoble.fr \\ 2 Laboratoire de Planétologie de Grenoble, UMR 5109-CNRS, Université Joseph Fourier, Grenoble, France
}

Received 19 January 2009 / Accepted 17 February 2009

\begin{abstract}
Context. For several reasons, methanol is believed to be formed on grain surfaces and, in warm environments, released in the gas phase. In the past, multiply deuterated isotopologues of methanol have been detected in gas phase around several low-mass protostars. In all these sources, there is significantly more $\mathrm{CH}_{2} \mathrm{DOH}$ than $\mathrm{CH}_{3} \mathrm{OD}$. Various hypotheses have been suggested to explain this anomaly, but none is fully convincing.

Aims. In this work, we test a new hypothesis experimentally: the spontaneous exchange between hydrogen and deuterium atoms in water ice as responsible for the deficiency of $\mathrm{CH}_{3} \mathrm{OD}$ with respect to $\mathrm{CH}_{2} \mathrm{DOH}$.

Methods. We follow the temperature dependence of the composition of interstellar ice analogs initially composed of $\mathrm{CD}_{3} \mathrm{OD}$ and $\mathrm{H}_{2} \mathrm{O}$. To this aim, thin films of intimate $\mathrm{H}_{2} \mathrm{O}: \mathrm{CD}_{3} \mathrm{OD}$ ice mixtures, condensed at low temperature $(<110 \mathrm{~K})$, are monitored by Fourier transform infrared (FTIR) spectroscopy up to the complete evaporation of $\mathrm{CD}_{3} \mathrm{OD}(\sim 170 \mathrm{~K})$.

Results. Rapid hydrogen/deuterium (H/D) exchange is observed, at $120 \mathrm{~K}$ and above, through the growth of the $v_{\mathrm{OD}}$ stretching mode of $\mathrm{HDO}$ at $\sim 2425 \mathrm{~cm}^{-1}$. It is also shown that H/D exchange occurs i) on the hydroxyl functional group of methanol, i.e through hydrogen bonds, and ii) before the completion of crystallization.

Conclusions. The present results suggest that the much lower abundance of $\mathrm{CH}_{3} \mathrm{OD}$ compared to $\mathrm{CH}_{2} \mathrm{DOH}$ in low-mass protostars could reflect H/D exchanges in water ice either prior to or definitely during the grain mantle sublimation. This solid-state depletion mechanism, so far neglected in the astronomical literature, might affect other deuterated molecules with hydrogen bonds.
\end{abstract}

Key words. molecular data - molecular processes - ISM: molecules

\section{Introduction}

A variety of interstellar molecules are highly enriched in deuterium. This well-known phenomenon, first discovered by Jefferts et al. (1973), was soon interpreted (Wilson et al. 1973) as occurring at a low temperature because of the differences in zero-point energies between deuterated and nondeuterated species. Recent observations towards regions of high density and heavy $\mathrm{CO}$ depletion, such as prestellar cores, have revealed that the gas-phase abundances of singly deuterated molecules are typically four orders of magnitude higher than the cosmic atomic D/H ratio of $1.5 \times 10^{-5}$ (Linsky 2003). Even more spectacular, doubly and triply deuterated molecules have been observed with $\mathrm{D} / \mathrm{H}$ ratios reaching $\sim 30 \%$ for $\mathrm{D}_{2} \mathrm{CO}$ (Ceccarelli et al. 1998; Loinard et al. 2002) and $\sim 3 \%$ for $\mathrm{CD}_{3} \mathrm{OH}$ (Parise et al. 2004). It should be noted that this extreme deuteration has been observed only in low-mass (solar-type) prestellar cores and protostars (Ceccarelli et al. 2007). A theoretical point of view has shown (Roberts et al. 2003) that the multiply deuterated isotopologues of $\mathrm{H}_{3}^{+}$efficiently transfer deuterons to other neutral molecules in very cold $(\leq 20 \mathrm{~K})$ gas depleted of its $\mathrm{CO}$ (because frozen onto the grain mantles). At the same time, the dissociative recombination of the deuterated ions leads to significant enhancement of the atomic $\mathrm{D} / \mathrm{H}$ ratio $(>0.1)$. As a consequence, molecules formed in the gas phase (e.g., Roueff et al. 2007) or onto the grain surfaces by atom addition reactions (e.g., Tielens 1983) would present large enhancements of the D- over
$\mathrm{H}$-bearing molecules with respect to the cosmic $\mathrm{D} / \mathrm{H}$ abundance ratio.

In the case of methanol, in particular, recent experimental works have demonstrated that a purely gas-phase synthesis is unlikely to reproduce the observed abundances (see Garrod et al. 2006, and references therein), while the hypothesis of methanol formation on cold grain surfaces by successive hydrogenation of $\mathrm{CO}$ has apparently been confirmed (Nagaoka et al. 2005). Moreover, Nagaoka et al. (2005) were able to reproduce a level of deuterium fractionation of methanol comparable to observations, including multiply deuterated isotopologues. These authors have shown, in particular, that the deuteration proceeds (in their experiment) mainly via the H-D substitution in solid methanol rather than via the successive hydrogenation and deuteration of $\mathrm{CO}$. Furthermore, the non-detection of $\mathrm{CH}_{3} \mathrm{OD}$ in their experiment was claimed to be consistent with the much lower gas-phase abundance of $\mathrm{CH}_{3} \mathrm{OD}$ compared to $\mathrm{CH}_{2} \mathrm{DOH}$ in low-mass class 0 protostars (Parise et al. 2004, 2006). In these sources, the $\left[\mathrm{CH}_{2} \mathrm{DOH}\right] /\left[\mathrm{CH}_{3} \mathrm{OD}\right]$ ratio was indeed found to be unexpectedly large (e.g. $20 \pm 14$ in IRAS 16293-2422) compared to the value of 3 predicted by a statistical surface formation mechanism (Charnley et al. 1997). Alternatively, Osamura et al. (2004) have theoretically studied the post-evaporative gasphase chemistry of the deuterated methanol isotopologues. They show that pairs of isotopologues with deuterium at different sites (e.g. $\mathrm{CH}_{3} \mathrm{OD}$ and $\mathrm{CH}_{2} \mathrm{DOH}$ ) cannot be interconverted efficiently. They conclude that $\mathrm{CH}_{3} \mathrm{OD}$ could actually be destroyed 
in the gas phase, through protonation reactions, on a much faster time scale than $\mathrm{CH}_{2} \mathrm{DOH}$.

In this Letter, we test a new hypothesis that has so far not been considered in the astronomical literature to explain the observed selective deuteration of methanol: the solid-state hydrogen/deuterium (H/D) spontaneous exchange between deuterated methanol and water. Such exchanges have been studied for a long time using various experimental approaches in water ices (see e.g., Devlin \& Buch 2007, and references therein). In the present work, $\mathrm{H} / \mathrm{D}$ exchanges are investigated by Fourier transform infrared (FTIR) spectroscopy of $\mathrm{H}_{2} \mathrm{O}: \mathrm{CD}_{3} \mathrm{OD}$ ice mixtures. The experimental method is described in Sect. 2. The results are presented in Sect. 3 and discussed in Sect. 4. We conclude with Sect. 5.

\section{Method}

Experiments were performed at the Laboratoire de Planétologie de Grenoble. Thin films of pure ices or intimate molecular mixtures of $\mathrm{H}_{2} \mathrm{O}: \mathrm{CD}_{3} \mathrm{OD}$ were condensed at low temperature (30-110 K) onto a $\mathrm{KBr}$ window, located at the bottom of the cold finger of a helium closed cycle cryostat. A co-deposition system with two gas inlets injected both gases independently in the vacuum cell and ensured the lack of isotopic exchange prior condensation onto the cold window. The $\mathrm{CD}_{3} \mathrm{OD}$ had a purity of $99.8 \%$ and $\mathrm{H}_{2} \mathrm{O}$ of $99.9999 \%$. The temperature was monitored with a heating resistor located onto the window holder and a PID controller. The ultra-vacuum in the cell was typically $\sim 10^{-8}$ mbars, thanks to a turbomolecular pump. Film thickness was measured during deposition from the interference fringes of the transmitted and reflected beams of an He-Ne laser. Mid-infrared spectra $\left(4000-400 \mathrm{~cm}^{-1}\right)$ were collected with a NICOLET800 FTIR spectrometer, equipped with a GLOBAR source and a DTGS detector. The $\mathrm{H}_{2} \mathrm{O}: \mathrm{CD}_{3} \mathrm{OD}$ molar ratio in solid films was estimated from the ratio of the condensation rates of pure $\mathrm{H}_{2} \mathrm{O}$ and $\mathrm{CD}_{3} \mathrm{OD}$, assuming similar sticking coefficients $(\sim 1$ at these low temperatures). However, thin film deposition continued after stopping gas injection, because of the remaining adsorbed molecules in the gas inlets. The solid film probably exhibited a concentration gradient close to its surface exposed to vacuum, and the $\mathrm{CD}_{3} \mathrm{OD}$ concentration was probably not estimated accurately.

\section{Results}

Preliminary experiments consisted in condensing stacked thin films (thickness $\sim \mu \mathrm{m}$ ) of pure ices of $\mathrm{CD}_{3} \mathrm{OD}$ and $\mathrm{H}_{2} \mathrm{O}$. No $\mathrm{H} / \mathrm{D}$ exchange was observed, regardless of the temperature, duration, and experimental conditions of deposition. In contrast, rapid $\mathrm{H} / \mathrm{D}$ exchanges were observed in $\mathrm{H}_{2} \mathrm{O}: \mathrm{CD}_{3} \mathrm{OD}$ intimate molecular mixtures, as we show below.

Two significant experiments with intimate $\mathrm{H}_{2} \mathrm{O}: \mathrm{CD}_{3} \mathrm{OD}$ mixtures are presented in the following. In the first one, a thin film was condensed at $90 \mathrm{~K}$ with an $\mathrm{H}_{2} \mathrm{O}$ deposition rate of $0.15 \mu \mathrm{m} \cdot \mathrm{min}^{-1}$, a $\mathrm{CD}_{3} \mathrm{OD}$ deposition of $8 \times 10^{-3} \mu \mathrm{m} \cdot \mathrm{min}^{-1}$, hence a $\mathrm{CD}_{3} \mathrm{OD}$ concentration in $\mathrm{H}_{2} \mathrm{O}$ ice of $\sim 3 \%$. In this experiment, the film was monitored by FTIR spectroscopy up to the complete $\mathrm{CD}_{3} \mathrm{OD}$ evaporation in the range 90-170 K. Infrared spectra were found to exhibit the fundamental vibrational modes of amorphous water ice, $v_{1}$ and $v_{3}$ as a broad band at $\sim 3300 \mathrm{~cm}^{-1}, v_{2}$ as the asymmetric broad band peaking at $\sim 1700 \mathrm{~cm}^{-1}$, and the libration band $\nu_{\mathrm{L}}$ at $\sim 830 \mathrm{~cm}^{-1}$ (see Table 1). It should be noted that the low $\mathrm{CD}_{3} \mathrm{OD}: \mathrm{H}_{2} \mathrm{O}$ ratio required film thicknesses leading to saturation of the strong stretch
Table 1. The observed vibrational frequencies $\left(\mathrm{cm}^{-1}\right)$ of $\mathrm{H}_{2} \mathrm{O}, \mathrm{HOD}$, and $\mathrm{CD}_{3} \mathrm{OD}$ in the condensed $\mathrm{H}_{2} \mathrm{O}: \mathrm{CD}_{3} \mathrm{OD}$ thin films.

\begin{tabular}{ll}
\hline \hline Vibrational mode & Band position $\left(\mathrm{cm}^{-1}\right)$ \\
\hline$v_{1}\left(\mathrm{H}_{2} \mathrm{O}\right)$ & $\sim 3300$ \\
$v_{2}\left(\mathrm{H}_{2} \mathrm{O}\right)$ & $\sim 1700$ \\
$v_{3}\left(\mathrm{H}_{2} \mathrm{O}\right)$ & $\sim 3300$ \\
$v_{\mathrm{L}}\left(\mathrm{H}_{2} \mathrm{O}\right)$ & $\sim 830$ \\
$v_{2}+v_{\mathrm{L}}\left(\mathrm{H}_{2} \mathrm{O}\right)$ & $\sim 2250$ \\
$v_{\mathrm{OD}}(\mathrm{HOD})$ & 2425 \\
$v_{\mathrm{OD}}\left(\mathrm{CD}_{3} \mathrm{OD}\right)$ & $\sim 2500-2350$ \\
$v_{\mathrm{CD}}\left(\mathrm{CD}_{3} \mathrm{OD}\right)$ & $2250,2225,2080$ \\
$\delta_{\mathrm{CD}_{3}}\left(\mathrm{CD}_{3} \mathrm{OD}\right)$ & 1115,1065 \\
$v_{\mathrm{CO}}\left(\mathrm{CD}_{3} \mathrm{OD}\right)$ & 980 \\
\hline
\end{tabular}

and anti-stretch fundamental water bands. The H/D exchanges were thus traced within the spectral range $2700-2000 \mathrm{~cm}^{-1}$ only (see Fig. 1). In this region, the most intense spectral feature is the broad water combination vibrational mode $v_{2}+v_{\mathrm{L}}$, onto which are superimposed several modes of the $\mathrm{CD}_{3}$ functional group of $\mathrm{CD}_{3} \mathrm{OD}$ and, when present, the $v_{\mathrm{OD}}$ stretching mode of HDO at $\sim 2425 \mathrm{~cm}^{-1}$. Vibrational modes of $\mathrm{CD}_{3} \mathrm{OD}$ are observed at 2250 and $2225 \mathrm{~cm}^{-1}$ for the antisymmetric stretching modes of the $\mathrm{CD}_{3}$ methyl group and at $2080 \mathrm{~cm}^{-1}$ for the $\mathrm{CD}_{3}$ symetric stretching mode. The band of the stretching mode $v_{\mathrm{OD}}$ of $\mathrm{CD}_{3} \mathrm{OD}$ is found to merge with the broad $v_{2}+v_{\mathrm{L}}$ water ice combination band. This clearly reflects the presence of numerous $\mathrm{CD}_{3} \mathrm{OD}$ multimers interacting with each other by strong hydrogen bonding (Quirico \& Schmitt 1997a,b).

The first major result of this experiment is the presence, at $120 \mathrm{~K}$ and above, of the $v_{\mathrm{OD}}$ stretching mode of HDO at $\sim 2425 \mathrm{~cm}^{-1}$ (Fig. 1). We emphasize that this band was completely absent at lower temperatures, while it was found to grow rapidly at $120 \mathrm{~K}$ (within a few hours). We note that it cannot be assigned to monomeric $\mathrm{CD}_{3} \mathrm{OD}$ because it is observed prior methanol sublimation, as supported by the intensity of the methyl band at $2080 \mathrm{~cm}^{-1}$.

The presence of HDO in water ice after the complete sublimation of $\mathrm{CD}_{3} \mathrm{OD}$ (at $170 \mathrm{~K}$ ) definitely evidences the fact that a $\mathrm{H} / \mathrm{D}$ transfer occured between $\mathrm{CD}_{3} \mathrm{OD}$ and $\mathrm{H}_{2} \mathrm{O}$ in solid state. The second crucial result concerns the location of the H/D exchange: the intensity, position, and shape of the $\mathrm{CD}_{3}$ functional group at $2080 \mathrm{~cm}^{-1}$ is unaltered throughout the whole growth of the HDO $v_{\mathrm{OD}}$ band (Fig. 1). This demonstrates that proton transfer occurred on the hydroxyl group of $\mathrm{CD}_{3} \mathrm{OD}$, presumably through hydrogen bonding.

In the second experiment, a thin film was condensed at $110 \mathrm{~K}$, and the $\mathrm{CD}_{3} \mathrm{OD}$ concentration in $\mathrm{H}_{2} \mathrm{O}$ ice was $\sim 1 \%$. Time-resolved measurements were performed over $\sim 150 \mathrm{~h}$ in the temperature range $110-130 \mathrm{~K}$, with a better time resolution than in the first experiment. We had specific concerns with the control of the kinetics by crystallization, which is known to enhance molecular motion and to promote hydrogen bonding. As shown in Fig. 2 the spectra look slightly different from those of the first experiment. The first reason is that different $\mathrm{CD}_{3} \mathrm{OD}$ concentrations led to different size distributions of $\mathrm{CD}_{3} \mathrm{OD}$ clusters, resulting in variations in the spectral distribution over the $v_{\mathrm{OD}}\left(\mathrm{CD}_{3} \mathrm{OD}\right)$ region. We also suspect that an almost pure $\mathrm{CD}_{3} \mathrm{OD}$ film deposited onto the firmly deposited mixture, because of condensation of $\mathrm{CD}_{3} \mathrm{OD}$ adsorbed in gas inlets.

Here again, no H/D exchange was observed at temperatures lower than $120 \mathrm{~K}$. At $T=120 \mathrm{~K}$, the H/D exchange was significant after only $6 \mathrm{~h}$. At that time, the $v_{\mathrm{OD}}$ vibrational mode of 


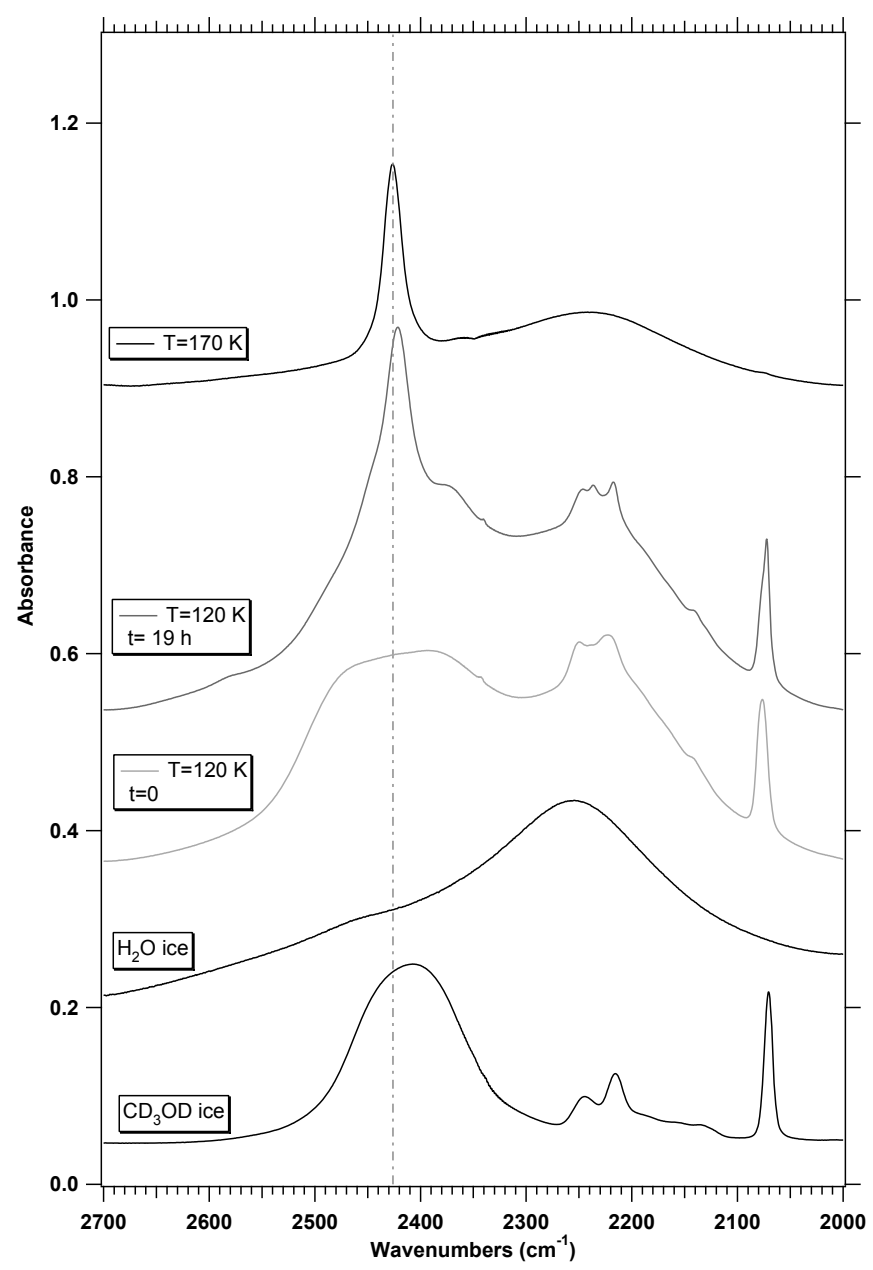

Fig. 1. First experiment (90-170 K). The isotopic exchange is monitored up to the complete evaporation of $\mathrm{CD}_{3} \mathrm{OD}$. The remaining crystalline (Ic) $\mathrm{H}_{2} \mathrm{O}$ ice film is enriched in HDO. At $120 \mathrm{~K}$, rapid $\mathrm{H} / \mathrm{D}$ exchange occurs. The experiment shows H/D exchange located on the hydroxyl functional group. Spectra are vertically offset to ensure clarity in the figure.

HDO appeared as a band peaking at $2425 \mathrm{~cm}^{-1}$, growing with increasing time and heating (left panel of Fig. 2). The librational water mode $v_{\mathrm{L}}$, which is a tracer of the crystalline state (Hagen \& Tielens 1982), was used to indicate that i) water ice has experienced the phase transition (Ial) $\rightarrow$ (Ic) at $120 \mathrm{~K}$ and that ii) this phase transition was not completed when the H/D exchange was significantly advanced (right panel of Fig. 2).

Finally, it should be noted that besides the increasing intensity of the HDO $v_{\mathrm{OD}}$ vibrational mode, in both experiments, complex spectral variations were observed in the spectra, including splitting, peak shifting, and variations in band intensities. It is not the purpose of this Letter to discuss those spectral variations in detail. They are definitely controlled by a variety of factors, in particular the degree of crystallinity and the degree of clustering of $\mathrm{CD}_{3} \mathrm{OD}$ molecules (Quirico \& Schmitt 1997a,b).

\section{Discussion}

The deuteration and the formation process of methanol are major issues in interstellar chemistry. Current gas-phase models do not account for the synthesis of methanol (Garrod et al. 2006). Therefore, formation routes onto and/or inside the icy mantles of interstellar grains have been proposed. Photolysis and radiolysis
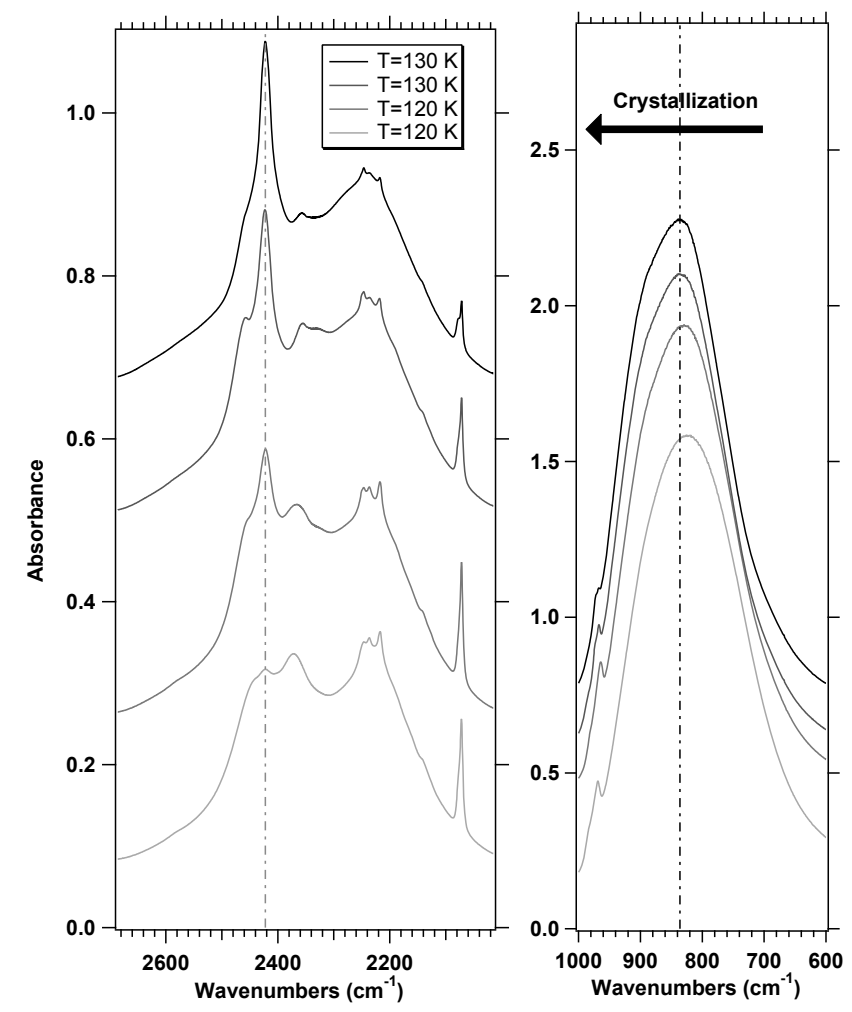

Fig. 2. Second experiment (110-130 K). Left: the isotopic H/D exchange between $\mathrm{H}_{2} \mathrm{O}$ and the hydroxyl functional group of $\mathrm{CD}_{3} \mathrm{OD}$ is traced by the HDO $v_{\mathrm{OD}}$ band at $2425 \mathrm{~cm}^{-1}$. Right: the libration water ice mode $v_{\mathrm{L}}$, used as a tracer of the crystalline state. H/D exchange occurs before the completion of crystallization. Spectra are vertically offset to ensure clarity in the figure.

experiments of interstellar ice analogs show that methanol is efficiently formed from $\mathrm{CH}_{4}: \mathrm{H}_{2} \mathrm{O}$ or $\mathrm{CO}: \mathrm{H}_{2} \mathrm{O}$ mixtures (Hudson \& Moore 1999; Wada et al. 2006). However, it is uncertain that such photolysis/radiolysis solid-state chemistry produces high $\mathrm{H} / \mathrm{D}$ fractionation. In particular, the $\mathrm{HDO} / \mathrm{H}_{2} \mathrm{O}$ ratio of water ice is very low (Parise et al. 2003; Dartois et al. 2003) and the deuteration of $\mathrm{CH}_{4}$ or $\mathrm{CH}_{3}^{+}$species is mostly unknown. Another possible chemical route is the reaction of $\mathrm{D}$ atoms at the surface of the grains, coming from a D-rich atomic gas produced by gasphase chemistry fractionation $(\mathrm{D} / \mathrm{H} \sim 0.1)$ (Watanabe \& Kouchi 2002; Nagaoka et al. 2005). As mentioned in the Introduction, the experiments of Nagaoka et al. (2005) have demonstrated that singly, doubly, and triply deuterated methanol isotopologues, with $\mathrm{D}$ atoms located in the methyl group, can be produced via H-D substitution in solid methanol.

Our experiments are complementary to these works, as they have focused on the loss of deuterium in ice mixtures, i.e. a solid-state depletion mechanism. They clearly demonstrate that $\mathrm{H} / \mathrm{D}$ exchange between the hydroxyl group of $\mathrm{CD}_{3} \mathrm{OD}$ and $\mathrm{H}_{2} \mathrm{O}$ necessarily occurs when ices are heated above $120 \mathrm{~K}$, during the crystallization of water. It should be noted that thermally activated $\mathrm{H} / \mathrm{D}$ exchange between $\mathrm{D}_{2} \mathrm{O}$ and $\mathrm{CH}_{3} \mathrm{OH}$ had been observed previously by Kawanowa et al. (2004) at temperatures above $140 \mathrm{~K}$, often described as a critical temperature. We also note that some controversy exists in the detailed mechanism of the H/D exchange (see e.g., Lee et al. 2007, and references therein). In our experiments, exchange was found to occur only in intimate molecular mixtures, i.e. in couples of molecules spatially closely related. We thus conclude that long scale proton 
diffusion is kinetically hampered at $T<170 \mathrm{~K}$ (at least on a laboratory time scale), and that the key role of crystallization is to promote mobility, hence connections among - $\mathrm{OH}$ functions through hydrogen bonds. Astrophysically, this H/D exchange might explain the relative abundances of deuterated isotopologues of methanol, at least in low-mass protostellar sources (Parise et al. 2004, 2006). It does not exclude the formation of OD methanol isotopologues on or in the grains, but suggests that $\mathrm{D}$ atoms located in the hydroxyl group of methanol should exchange with protons from ice. From this point of view, it is consistent with the presence of $\mathrm{CH}_{3} \mathrm{OD}$ species in star-forming regions, while these are undetected in the experiment of Nagaoka et al. (2005). Moreover, the level of deuteration of $\mathrm{H}_{2} \mathrm{CO}$ in lowmass protostars is similar to that of the methyl group of $\mathrm{CH}_{3} \mathrm{OH}$ (Parise et al. 2006). This is also consistent with that fact that, in contrast to $-\mathrm{OD}$ functions, $-\mathrm{CD}$ functions do not exchange with $\mathrm{H}_{2} \mathrm{O}$.

In astrophysical conditions, this general mechanism might occur at lower temperatures owing to long time scales. In $10^{5}$ years, the complete crystallization could occur at temperatures as low as $90 \mathrm{~K}$ (Schmitt 1992). Furthermore, proton mobility and thus isotopic exchanges may be triggered at even lower temperatures, without crystallization, during the progressive and partial structural reorganization of amorphous ice occurring during warmup above the condensation temperature (Schmitt et al. 1989). Definitely, systematic studies on $\mathrm{CD}_{3} \mathrm{OD}: \mathrm{H}_{2} \mathrm{O}$ ice mixtures are required to provide more quantitative data. Other factors may also play a key role. The $\mathrm{CH}_{3} \mathrm{OH}: \mathrm{H}_{2} \mathrm{O}$ ratio, which can be very high in some protostellar sources (Pontoppidan et al. 2003; Dartois et al. 1999), might limit the H/D exchange because $\mathrm{CH}_{3} \mathrm{OD}$ molecules could be protected by others and could therefore avoid close interactions with $\mathrm{H}_{2} \mathrm{O}$. Such segregation effects have been suggested by comparing spectra of massive protostars with laboratory data (Ehrenfreund et al. 1998). In contrast, other effects could promote the H/D exchange by producing free protons, e.g. photolysis, radiolysis or dopants (Johari 2002). Finally, since this depletion mechanism occurs through the hydrogen bond, other detected molecules like deuterated isotopologues of ammonia could also exchange deuterium atoms with water ice if they experience some residence time on icy grains.

\section{Conclusions}

In this Letter, thin films of intimate $\mathrm{H}_{2} \mathrm{O}: \mathrm{CD}_{3} \mathrm{OD}$ ice mixtures condensed at a low temperature $(<110 \mathrm{~K})$ have been investigated by FTIR spectroscopy up to the complete evaporation of $\mathrm{CD}_{3} \mathrm{OD}$ $(\sim 170 \mathrm{~K})$. It has been demonstrated that i) rapid H/D exchange between solid $\mathrm{H}_{2} \mathrm{O}$ and $\mathrm{CD}_{3} \mathrm{OD}$ occurs at temperatures as low as $120 \mathrm{~K}$ and before the completion of crystallization and ii) $\mathrm{H} / \mathrm{D}$ exchange takes place on the hydroxyl functional group of methanol, through hydrogen bonds. These results suggests that the much lower abundance of $\mathrm{CH}_{3} \mathrm{OD}$ compared to $\mathrm{CH}_{2} \mathrm{DOH}$ in low-mass class 0 protostars might simply reflect H/D spontaneous exchange either prior to or definitely during the grain mantle sublimation. This solid-state depletion mechanism, so far neglected in the astronomical literature, might affect other deuterated molecules with hydrogen bonds (e.g. $\mathrm{ND}_{3}, \mathrm{~N}_{2} \mathrm{D}^{+}$). This will be explored in future works, both in the laboratory and at the telescope.

Acknowledgements. One of us (A.R.) is supported by a fellowship of the Ministère de l'Enseignement Supérieur et de la Recherche. Support by the French National Research Agency (ANR-08-BLAN-0225-01) is acknowledged.

\section{References}

Ceccarelli, C., Castets, A., Loinard, L., Caux, E., \& Tielens, A. G. G. M. 1998, A\&A, 338, L43

Ceccarelli, C., Caselli, P., Herbst, E., Tielens, A. G. G. M., \& Caux, E. 2007, in Protostars and Planets V, ed. B. Reipurth, D. Jewitt, \& K. Keil, 47

Charnley, S. B., Tielens, A. G. G. M., \& Rodgers, S. D. 1997, ApJ, 482, L203

Dartois, E., Schutte, W., Geballe, T. R., et al. 1999, A\&A, 342, L32

Dartois, E., Thi, W.-F., Geballe, T. R., et al. 2003, A\&A, 399, 1009

Devlin, J. P., \& Buch, V. 2007, J. Chem. Phys., 127, 091101

Ehrenfreund, P., Dartois, E., Demyk, K., \& D’Hendecourt, L. 1998, A\&A, 339, L17

Garrod, R., Park, I. H., Caselli, P., \& Herbst, E. 2006, Faraday Discuss., 133, 51 Hagen, W., \& Tielens, A. G. G. M. 1982, Spectrochim. Acta Part A, 38, 1089

Hudson, R. L., \& Moore, M. H. 1999, Icarus, 140, 451

Jefferts, K. B., Penzias, A. A., \& Wilson, R. W. 1973, ApJ, 179, L57

Johari, G. P. 2002, J. Chem. Phys., 117, 2782

Kawanowa, H., Kondon, M., Gotoh, Y., \& Souda, R. 2004, Surface Sci., 566, 1190

Lee, C.-W., Lee, P.-R., Kim, Y.-K., \& Kang, H. 2007, J. Chem. Phys., 127

Linsky, J. L. 2003, Space Sci. Rev., 106, 49

Loinard, L., Castets, A., Ceccarelli, C., et al. 2002, Planet. Space Sci., 50, 1205

Nagaoka, A., Watanabe, N., \& Kouchi, A. 2005, ApJ, 624, L29

Osamura, Y., Roberts, H., \& Herbst, E. 2004, A\&A, 421, 1101

Parise, B., Castets, A., Herbst, E., et al. 2004, A\&A, 416, 159

Parise, B., Ceccarelli, C., Tielens, A. G. G. M., et al. 2006, A\&A, 453, 949

Parise, B., Simon, T., Caux, E., et al. 2003, A\&A, 410, 897

Pontoppidan, K. M., Dartois, E., van Dishoeck, E. F., Thi, W.-F., \& d'Hendecourt, L. 2003, A\&A, 404, L17

Quirico, E., \& Schmitt, B. 1997a, Icarus, 128, 181

Quirico, E., \& Schmitt, B. 1997b, Icarus, 127, 354

Roberts, H., Herbst, E., \& Millar, T. J. 2003, ApJ, 591, L41

Roueff, E., Herbst, E., Lis, D. C., \& Phillips, T. G. 2007, ApJ, 661, L159

Schmitt, B. 1992, in Interrelations between Physics and Dynamics for Minor Bodies in the Solar System, ed. D. Benest, \& C. Froeschl, 265

Schmitt, B., Grim, R. J. A., \& Greenberg, J. M. 1989, in Infrared Spectroscopy in Astronomy, Proc. 22nd Eslab Symposium, ed. D. Benest, \& C. Froeschl, ESA Special Publ., 290, 213

Tielens, A. G. G. M. 1983, A\&A, 119, 177

Wada, A., Mochizuki, N., \& Hiraoka, K. 2006, ApJ, 644, 300

Watanabe, N., \& Kouchi, A. 2002, ApJ, 571, L173

Wilson, R. W., Penzias, A. A., Jefferts, K. B., \& Solomon, P. M. 1973, ApJ, 179, L107 